\title{
Lectures des enquêtes sociologiques : les processus d'interprétation du point de vue du théologien pratique
}

\author{
Les numéros correspondant à la pagination de la version imprimée sont placés entre crochets \\ dans le texte et composés en gras.
}

\begin{abstract}
Isabelle Grellier
Faculté de Théologie protestante, Université de Strasbourg, Professeure en théologie
\end{abstract} pratique

<591> Résumé : Pour la théologie pratique qui ne peut faire l'économie d'un travail de terrain, l'enquête quantitative peut être un outil précieux. Mais une telle tâche - qui relève d'abord de la sociologie - nécessite certaines règles. Le théologien pratique devra en particulier être vigilant à ce que la dimension normative qui n'est pas absente de sa discipline n'empiète pas sur l'élaboration de l'enquête et sur l'interprétation des données recueillies. Il lui faudra par ailleurs mener à bien le travail de corrélation qui est au cœur de la démarche de théologie pratique avant de chercher à traduire les résultats de l'enquête en décisions concrètes. L'écart entre la réalité et ce que nous voudrions qu'elle soit ne pourrait-il pas être fructueux pour la réflexion?

Mots-clefs : théologie pratique - enquête quantitative - interprétation - corrélation normativité

Nécessairement arrimée à une analyse des réalités, la théologie pratique est souvent amenée à se servir d'un travail de terrain pour nourrir sa réflexion. Observation participante, rechercheaction, entretiens qualitatifs, enquêtes quantitatives, etc., ce travail de terrain peut prendre des formes diverses, selon les objectifs visés et les moyens disponibles. C'est l'enquête quantitative que nous privilégierons ici - même si plusieurs des indications qui seront données, en particulier sur la démarche d'interprétation, peuvent valoir pour d'autres formes de recherche.

Construire une problématique, élaborer un questionnaire, effectuer un échantillon, mener à bien la récolte des données, interpréter celles-ci et en tirer des enseignements en termes de théologie pratique..., telles sont les étapes principales d'un travail d'enquête, et chacune constitue une activité complexe, qui ne peut s'improviser. C'est à donner quelques éléments de réflexion sur cette démarche que ma contribution voudrait s'attacher.

$<$ 592> Elle vient en complément des indications données par Christophe Monnot dans cet ouvrage, car la plupart des étapes relèvent d'une démarche sociologique. C'est l'occasion de préciser combien il est précieux, pour le théologien pratique, de pouvoir s'appuyer, pour mener à bien un travail d'enquête, sur les compétences de sociologues et, mieux encore, de pouvoir 
collaborer avec eux tout au long de la démarche. Car le théologien pratique ne possède que rarement les compétences nécessaires pour une enquête sociologique ; et même dans le cas où il les aurait, il est compliqué pour une seule personne de tenir les deux positionnements épistémologiques, celui du sociologue et celui du théologien ${ }^{1}$. Or la différence de regard enrichit la réflexion. Concrètement, le sociologue peut aider le théologien pratique à ne pas traduire trop hâtivement les données recueillies en décisions concrètes, sans avoir fait le détour de l'interprétation sociologique et de l'évaluation théologique.

Je voudrais dans un premier temps revenir à la démarche de théologie pratique pour expliciter son rapport au travail de terrain. Je soulignerai ensuite quelques points spécifiques à une enquête menée avec une visée de théologie pratique, ceci en m'appuyant sur la contribution de Christophe Monnot. La troisième partie, davantage nourrie d'exemples, sera consacrée au processus d'analyse et d'interprétation des données recueillies, étape indispensable avant un retour à la pratique. Je reviendrai en conclusion sur une question qui traversera toutes ces lignes, celle du rapport à la norme qui est si différent en sociologie et en théologie pratique.

\section{Pourquoi des ENQuêtes EN THÉOlogie PRATIQUe ? RETOUR SUR LA DÉMARCHE de THÉOLOGIE PRATIQUE}

On a beaucoup cherché, depuis quelques décennies, à théoriser la démarche de théologie pratique et ce travail est toujours à refaire, car on ne peut l'enfermer dans des règles de fonctionnement trop étroites. Bien davantage qu'une technique pour laquelle on pourrait élaborer des processus reproductibles d'une situation à l'autre, il s'agit d'un artisanat ${ }^{2}$, très dépendant des lieux et conditions de production.

<593> Une conviction cependant fait l'unanimité aujourd'hui : la théologie pratique ne peut se contenter d'être la science d'application d'affirmations théoriques issues de l'exégèse ou de la dogmatique ; elle ne peut non plus être simplement théorisation d'expériences pratiques. Mais il lui faut sans arrêt réinventer une démarche de corrélation ${ }^{3}$ entre la théorie et la pratique, entre les affirmations de la tradition chrétienne et les réalités du monde contemporain. Dans les sociétés occidentales où la sécularisation a abouti à « l'exculturation ${ }^{4}$ du christianisme, cette démarche représente un défi à la fois considérable et incontournable si l'on veut que le message chrétien ait des chances de parler à nos contemporains.

\footnotetext{
${ }^{1}$ On pourra se référer à divers articles de Jean Joncheray, sociologue ayant souvent travaillé de concert avec des théologiens pratiques. Voir par exemple Jean JONCHERAY, «Théologie et sciences humaines », in Gilles Routhier et Marcel Viau (dir.), Précis de Théologie pratique, Montréal/Bruxelles, Novalis/Lumen Vitae, 2004, p.167-178.

${ }^{2}$ Ce terme a été particulièrement mis à l'honneur par Jacques AUDINET, qui a été professeur à l'Institut catholique de Paris et à l'Université de Metz et qui a beaucoup contribué à l'adoption du vocable «théologie pratique » dans le monde catholique. Voir par exemple ses Ecrits de théologie pratique (Québec/Paris/Bruxelles/Genève, Novalis/Cerf/Lumen Vitae/Labor et Fides, 1995), et en particulier son chapitre «De l'artisanat théologique », p. 165-175.

${ }^{3}$ Ce terme que l'on doit en particulier au théologien Paul Tillich se trouve explicité dans la plupart des ouvrages traitant de la démarche de théologie pratique. Voir par exemple Marc DuMAS, "Corrélation - Tillich et Schillebeeckx » in Précis de Théologie pratique (op. cit., p.71-83), ou Elisabeth PARMENTIER, "La corrélation. Des modèles, leurs chances et leurs limites », in La Théologie pratique. Analyses et prospectives, (sous la direction d'E. Parmentier, Strasbourg, Presses Universitaires, 2008, p. 69-87).

${ }^{4}$ Pour reprendre le terme si juste qu'emploie la sociologue Danièle HerviEu-LÉGER dans son ouvrage Catholicisme, la fin d'un monde (Paris, Bayard, 2003)
} 
Dans une telle démarche, il s'agit, comme le dit Marc Dumas, de « conserver l'autonomie des deux pôles, tout en insistant sur leur dépendance mutuelle $»^{5}$. « Dépendance mutuelle » : le terme est important dans une démarche croyante. Bien évidemment le croyant considère que le message chrétien constitue, pour le monde d'aujourd'hui comme pour celui d'hier, une richesse à recevoir qui peut donner sens à nos vies, individuellement et collectivement, en Église - bien sûr - et plus globalement dans la société. Les pratiques ecclésiales comme les pratiques sociales des chrétiens doivent se nourrir des réflexions théologiques. Mais réciproquement, que serait une pastorale - et plus largement que serait une théologie chrétienne - qui ne serait enracinée dans les expériences de vie des hommes et des femmes de notre temps ? Comment une théologie qui se réfère au Verbe habitant notre humanité pourrait-elle être fidèle à son fondement si elle ne se mettait réellement à l'écoute de tous ceux, croyants ou non, à qui l'Évangile est destiné, qui peut-être en vivent ou peut-être en sont porteurs sans le savoir. C'est donc bien une interpellation réciproque qu'il s'agit de mettre en œuvre.

C'est dire qu'une recherche en théologie pratique se nourrit presque toujours d'un travail de terrain. Les objectifs peuvent en être divers, et il est important de bien clarifier ce que l'on en attend avant de se lancer dans un travail d'enquête.

On peut chercher à mieux connaître la réalité des pratiques ecclésiales dans tel domaine - la catéchèse, la liturgie, etc. - , pour savoir ce qui se fait $<\mathbf{5 9 4}>$ effectivement dans les Églises locales, pour comprendre ce qui «marche » bien, moins bien, ou pas du tout et favoriser un partage d'expériences, éventuellement même pour vérifier la conformité de ces pratiques locales aux décisions synodales... Les personnes interrogées seront alors plutôt les responsables des paroisses et autres lieux d'Église.

On peut aussi chercher à connaître les opinions et les attentes des membres d'Église - ou, selon l'objectif de l'enquête, de personnes extérieures aux Églises - pour proposer des pratiques qui « collent » mieux à leurs besoins et sensibilités.

De telles perspectives, que l'on peut qualifier d'utilitaristes, sont bien sûr intéressantes dans une visée purement pratique, mais elles resteront en retrait de ce que l'on peut attendre d'une recherche en théologie pratique si elles ne font pas le détour par une réflexion théologique approfondie sur les enjeux de ces pratiques. On pourrait dire, d'une certaine façon, que ce type d'enquête est à une véritable démarche de théologie pratique ce qu'une enquête sur la consommation menée par une entreprise cherchant à adapter ses produits aux attentes des consommateurs serait à une enquête de sociologie. Or une stratégie d'Église, une pastorale, des pratiques ecclésiales ne peuvent simplement être la transposition des désirs des "consommateurs"; leur élaboration exige le passage par une analyse théologique. Réciproquement, d'ailleurs, la théologie elle-même y serait perdante dans la mesure où un des enjeux de la théologie pratique est de poser des questions nouvelles à la théologie ou d'aider à l'interroger différemment.

\section{LA DÉMARCHE DE L'ENQUÊTE}

Ch. Monnot décrit fort bien les différentes étapes d'une enquête, différentes étapes qui sont aussi nécessaires pour une recherche en théologie pratique, et je vais me contenter d'insister sur certains points ou d'ajouter quelques remarques plus spécifiques à notre domaine.

\footnotetext{
${ }^{5}$ M .DUMAS, « Corrélation - Tillich et Schillebeeckx », art. cit., p. 82.
} 


\section{La problématique :}

Il faut d'abord redire ici la nécessité d'élaborer une véritable problématique, sans laquelle un travail d'enquête n'aurait pas de sens. Formuler précisément le questionnement qui est au cœur du travail, former des hypothèses que l'enquête pourra confirmer ou infirmer : la théologie pratique ne saurait faire l'économie de ces étapes constitutives de tout travail scientifique. Trop souvent on se surprend au moment de l'interprétation à regretter de n'avoir pas posé <595> telle question qui aurait été utile ... À vrai dire, on ne pourra sans doute jamais éviter complétement cela, mais à se précipiter dans une enquête sans avoir correctement formulé sa problématique est un moyen sûr de préparer des regrets !

La construction de la problématique se fait souvent par tâtonnements et cela passe aussi bien par des lectures des travaux existants que par une première observation du terrain à étudier. Ceci pour permettre à la fois d'élargir son propre questionnement et de le préciser.

Il faudra aussi, dans cette étape préalable à la construction de l'enquête, prendre connaissance des enquêtes déjà réalisées, qui peuvent offrir une mine de données susceptibles de nourrir la réflexion. Il s'agira alors de construire sa propre enquête en complémentarité avec ce qui existe déjà, au plus près de sa propre problématique ; parfois même, on s'apercevra qu'une nouvelle enquête quantitative n'est pas indispensable et qu'il est plus judicieux de compléter la récolte des données par quelques entretiens dans une démarche qualitative.

\section{Se servir de l'existant :}

Nous avons en effet la chance en Europe de disposer d'un certain nombre d'enquêtes sociologiques menées dans les règles de l'art par des personnes qui sont généralement bien plus équipées pour ce type de travail que des théologiens pratiques.

Un premier avantage est tout simplement qu'il y a là à disposition des informations nombreuses, le plus souvent gratuites ${ }^{6}$, que le chercheur n'a pas eu à collecter (et qu'il n'aurait, la plupart du temps, pas eu les moyens matériels et financiers de rassembler !). Certes, ces données n'ont pas été construites en fonction de sa problématique spécifique. Et il pourra se trouver frustré de la formulation de telle ou telle question qui ne lui permet pas bien de vérifier ses hypothèses, ou de l'absence de telle autre qui lui aurait été utile. Mais ces inconvénients peuvent présenter aussi des avantages ; car telle question ou telle formulation auxquelles il n'aurait pas pensé peuvent aider le chercheur à sortir de ses a priori et à ouvrir son horizon. Menées par des personnes plus extérieures aux Églises, ces enquêtes ont moins tendance à utiliser la « langue de bois » religieuse qui peut ne pas parler aux personnes interrogées. Par ailleurs elles offrent souvent la possibilité d'une comparaison entre les opinions ou attitudes des croyants et celles de la population globale. Or c'est à travers la comparaison que les chiffres parlent le mieux.

$<$ 596 $>$ Ces enquêtes préexistantes peuvent être grosso modo de deux sortes. Il y a celles qui s'intéressent directement aux questions religieuses ; dirigées par des sociologues des religions dans le cadre de leurs travaux scientifiques, souvent avec le soutien d'institutions et de journaux religieux, elles s'attachent à analyser les croyances, les pratiques religieuses et éthiques, et/ou les opinions d'une population en mettant au centre le critère religieux; et certaines affinent l'étude en interrogeant spécifiquement une population précise : les catholiques, les musulmans, les protestants par exemple. Ainsi les protestants français ont fait l'objet, en 2010, d'une

\footnotetext{
${ }^{6}$ On trouve souvent sur les sites des instituts de sondage les résultats complets de ces enquêtes.
} 
enquête importante menée par l'IFOP ${ }^{7}$ - enquête qui peut s'enrichir d'une comparaison, mutatis mutandis ${ }^{8}$, avec des enquêtes antérieures (1995 et 2006) menées auprès de personnes se disant " proches du protestantisme », ce qui permet de mesurer les évolutions dans le temps.

On peut associer à cette catégorie les larges enquêtes sur les valeurs, menées régulièrement au niveau européen, qui comportent toujours un volet sur les appartenances et croyances religieuses 9 .

Il y a par ailleurs des enquêtes qui n'abordent les questions religieuses qu'indirectement, comme une des variables pouvant influer sur la thématique étudiée ; à travers les tris croisés en fonction de la variable religieuse, elles fournissent des indications comparatives sur les opinions ou pratiques des personnes relevant de telle religion par rapport à la population globale.

On trouvera ci-dessous, dans le paragraphe consacré à l'interprétation des données, quelques exemples relevant de ces différentes catégories.

\section{La rédaction d'un questionnaire}

Cette étape exige une très grande vigilance, le vocabulaire employé pouvant recéler des pièges.

Sauf questionnement particulier - quand on veut savoir par exemple si tel vocable théologique est reçu ou non -, il s'agit d'éviter le plus possible tout jargon d'Église. L'objectif est d'abord, bien sûr, de permettre que les questions soient vraiment intelligibles par toutes les personnes interrogées, y compris par celles qui sont distanciées des Églises; d'autant plus que des questions $<\mathbf{5 9 7}>$ incompréhensibles décourageraient les personnes sollicitées de répondre à l'enquête.

Mais un vocabulaire trop marqué recèle un autre piège : celui de susciter - souvent inconsciemment chez les personnes interrogées - des réponses qui ne seraient pas leur opinion, mais celles qu'ils imagineraient devoir donner en tant que " bons chrétiens »; un vocabulaire et des questionnements trop proches de ceux que les répondants ont pu entendre au catéchisme risquent de donner des réponses convenues. À moins qu'au contraire, le renvoi ainsi opéré à la culture ecclésiale ne suscite des réactions allergiques chez ceux qui ont des comptes à régler avec les Églises, les empêchant, tout autant, de répondre en liberté.

Il sera important aussi de veiller à l'intitulé du questionnaire - lequel peut orienter les réponses. Prenons ici un exemple qui interroge d'ailleurs au-delà des simples questions de vocabulaire : le Centre de sociologie du protestantisme de la Faculté de théologie protestante de Strasbourg avait organisé il y a quelques années une enquête sur la prédication, dont le support était un questionnaire passé lors d'un culte d'un dimanche ordinaire dans quelques paroisses protestantes alsaciennes ${ }^{10}$. Le moment retenu - juste après une prédication, bonne ou moins bonne -, ainsi que l'intitulé du questionnaire - "enquête sur la prédication»induisaient nécessairement des biais dont il a fallu tenir compte lors de l'interprétation ; pour s'arrêter au deuxième élément, les répondants auraient-ils été aussi nombreux à dire que la

\footnotetext{
${ }^{7}$ On trouvera l'ensemble des résultats chiffrés sur le site de l'IFOP, et une analyse de cette enquête dans Sébastien FATH et Jean-Paul WILlAIME (dir.), La nouvelle France protestante. Essor et recomposition au XXIe siècle, Genève, Labor et Fides, 2011.

${ }^{8}$ La prudence est nécessaire, car les enquêtes de 1995 et 2006 prenaient en compte aussi les opinions de personnes catholiques ou non-croyantes qui avaient choisi le protestantisme comme la religion dont ils se sentaient « le plus proche » parmi une liste de religions (la réponse 'aucune’ leur était bien sûr aussi proposée).

${ }^{9}$ Voir par exemple le site http://www.europeanvaluesstudy.eu/. Ces enquêtes ont lieu tous les neuf ans depuis 1990.

${ }^{10}$ On trouvera une analyse de cette enquête dans Isabelle GrELLIER, « Attentes à l'égard de la prédication et écoute de celle-ci dans diverses paroisses alsaciennes », Foi et Vie, Volume LXXXV, n 2-3, 1986, p. 115-142.
} 
prédication était très importante pour eux, voire indispensable, si l'intitulé du questionnaire avait mis en avant le culte plutôt que la prédication?

Dans cette étape d'élaboration d'un questionnaire aussi, il sera utile de se référer aux travaux existants. Reprendre des questions déjà posées dans des enquêtes antérieures - à condition bien sûr d'une part que ces questions s'inscrivent dans la problématique étudiée, d'autre part que leur formulation puisse encore parler aux contemporains - est un outil précieux pour pouvoir mesurer des évolutions dans le temps.

\section{INTERPRÉTER}

Une fois les données collectées, il s'agit bien sûr de les interpréter - une tâche essentielle sans laquelle l'enquête resterait lettre morte, et qui est pourtant parfois négligée, la tentation étant grande de traduire immédiatement les données recueillies en décisions concrètes.

<598> «Interpréter », qu'est-ce à dire ? Le verbe apparaît dans la langue française dès le $\mathrm{XII}^{\mathrm{e}}$ siècle avec le sens « expliquer, éclaircir », « traduire ${ }^{11}$. Il s'agit d'une tâche de médiation - et c'est bien ce rôle que doit jouer l'interprétation d'une enquête entre la collecte de données et la réflexion théologique, suivie éventuellement de l'élaboration de stratégies ecclésiales.

Il est amusant de constater qu'à la fin du XIV , le nom « interprète » désigne « celui ou celle qui transmet et explique la volonté des dieux $»^{12}$. Et si l'on en croit l'ouvrage de P.-L. Dubied ${ }^{13}$, c'est justement la vocation du pasteur que d'être un interprète. Il ne faut pas pour autant s'imaginer trop vite que l'enquête nous mettrait en capacité de discerner et de transmettre « la volonté des dieux »! Il s'agit plutôt d'essayer d'entendre et de comprendre les opinions, compréhensions et souhaits des personnes interrogées qui malgré le proverbe « vox populi, vox $d e i »$ ne sont pas nécessairement conformes à la volonté de Dieu... Et c'est dans un deuxième temps du travail d'interprétation, temps plus spécifique à la théologie pratique, qu'on essayera de confronter cette vox populi à ce que l'on peut essayer, comme théologien, de discerner de la vox dei.

Il s'agit de rester d'autant plus prudent que l'interprétation laisse toujours place, on le sait bien, à une certaine subjectivité. Et c'est l'occasion de redire combien il est précieux de mener cette tâche d'interprétation en dialogue entre plusieurs personnes ayant des points de vue différents sur la thématique de l'enquête. Une collaboration sociologue-théologien pratique est ici encore particulièrement recommandée; et il peut être intéressant d'y adjoindre l'un ou l'autre acteur de terrain capable d'expliciter certaines des caractéristiques du lieu.

\section{Analyser les données et se laisser interroger par elles}

Le travail d'analyse des données recueillies sera celui de toute enquête de sociologie. Que disent les chiffres en valeur absolue ? Quels sont les critères qui influent le plus sur les positions des répondants : leur âge ? leur appartenance sociale ? leur genre ? leur statut familial ? Quelles sont - si on a les moyens de les percevoir - les évolutions dans le temps sur les thématiques envisagées ? Et, bien sûr, comment peut-on comprendre ces données ? Quelles questions posent-elles ? Viennent-elles confirmer ou non les hypothèses que l'on avait formées ?

<599> On l'aura compris, la comparaison donne toujours particulièrement à penser. Ce qui signifie qu'il faudra examiner attentivement les tris croisés, qui explicitent les réponses

${ }^{11}$ Voir Alain REY (dir.), Dictionnaire historique de la langue française Le Robert, Paris, Dictionnaire Le Robert, 1992, t.1, p. 1043

12 Ibid.

${ }^{13}$ Voir Pierre-Luigi DubIED, Le pasteur : un interprète, Genève, Labor et Fides, coll « Pratiques n³ », 1990 
spécifiques de telle ou telle catégorie de population, en fonction de l'âge, de l'appartenance sociale, de la pratique cultuelle, de l'orientation théologique etc.

On a l'habitude en protestantisme de prendre assez bien en compte les variables d'âge ou de sexe, et nous sommes assez conscients du fait que ces réalités influent sur nos positions. Par contre - et sans doute parce que nous sommes marqués par une anthropologie qui privilégie l'individu plus que l'appartenance à un groupe, nous sous-estimons parfois l'effet des appartenances sociales. C'est une des richesses d'une enquête menée dans les règles de l'art qui fera donc place à des questions sur les diplômes et la situation professionnelle des répondants - que de nous rappeler l'importance de ces réalités sociales, y compris dans l'élaboration d'une pastorale.

Les traitements des données par les logiciels ad hoc font apparaître que certaines différences sont significatives tandis que d'autres ne le sont pas (soit qu'elles soient très faibles, soit qu'elles soient construites sur des effectifs de répondants trop petits). Il faudra donc bien sûr s'attacher aux différences significatives. Quand on effectue un traitement manuel, il faut traduire le nombre de répondants de chaque catégorie en pourcentages ; les différences pourront être jugées significatives si, pour un nombre de répondants suffisamment grand (au moins une vingtaine ou une trentaine de personnes dans la sous-catégorie envisagée), les pourcentages diffèrent d'au moins 6 ou $8 \%{ }^{14}$. -

Prenons quelques exemples pour expliciter mieux la démarche.

Pour qui accompagne des malades ou réfléchit à une pastorale de la santé, ou pour qui s'intéresse à l'œcuménisme intra-protestant, les réponses à la question « en cas de maladies, comptez-vous sur des guérisons miraculeuses? » posée dans l'enquête IFOP de 2010 peuvent donner à réfléchir. Sur l'ensemble des répondants, $7 \%$ choisissent la réponse, « oui, si je me suis bien conduit devant Dieu », $24 \% \%$ « oui, quel qu'ait été mon comportement devant Dieu » (soit $31 \%$ de oui), $20 \%$ répondent « non » et $48 \%$ «non, jamais » (68\% de non). Parmi les répondants de sensibilité luthéro-réformée, $13 \%$ répondent oui et $87 \%$ choisissent le non, alors que les répondants de sensibilité émergente (évangéliques, pentecôtistes, charismatiques...) sont $70 \%$ à retenir le oui (dont $56 \%$, « oui, quel qu'ait été mon comportement devant Dieu ») et $28 \%$ à répondre non. Ces chiffres ne sont pas forcément très surprenants pour qui connaît un peu les diverses sensibilités du monde protestant, mais ils permettent de mesurer le fossé qui sépare les différents courants.

$<600>$ Autre exemple, issu lui aussi d'une enquête centrée sur les questions religieuses, le sondage CSA mené en 1995 auprès de personnes se disant proches du protestantisme. Celui-ci révélait que 32\% des luthériens ou des personnes proches des Églises luthériennes disaient croire « tout à fait » au salut par la grâce seule, pour $42 \%$ des réformés (ou des proches du courant réformé), et $65 \%$ des personnes se reconnaissant plutôt dans les courants évangéliques ${ }^{15}$. Ces chiffres peuvent étonner car l'on sait bien que c'est Luther et la tradition luthérienne qui ont le plus mis l'accent sur cette affirmation, alors que le monde évangélique donne une grande importance à la sanctification. Comment comprendre ce décalage ? Une piste d'explication peut résider dans le fait que les personnes qui se réclament des courants évangéliques relèvent davantage que les autres répondants d'un protestantisme de conversion (ce que montrent par ailleurs les enquêtes), ce qui les fait adhérer plus fortement aux références du groupe. On peut aussi faire l'hypothèse que se situant davantage que les luthéro-réformés en rupture avec leur environnement culturel et relationnel, ces personnes proches des courants évangéliques sont amenées à mieux savoir dire ce qu'est leur croyance. Réciproquement on peut penser que quelqu'un qui a grandi dans un village majoritairement luthérien comme il en restait en Alsace il y a quelques décennies avait moins besoin de savoir définir ce qu'il croyait

\footnotetext{
${ }^{14}$ Plus les effectifs sont faibles, et plus l'écart entre les pourcentages doit être grand pour être significatif.
}

${ }^{15}$ Sondage CSA de 1995 (ces questions n'ont pas été reprises dans les sondages ultérieurs). 
(ou ce que son groupe croyait), car il avait finalement peu de raisons d'être interpelé sur ses croyances. C'est donc le poids des réalités sociales que ces chiffres refléteraient, au moins en partie.

Le dernier exemple est issu d'une enquête récente sur l'infidélité féminine ${ }^{16}$. La thématique n'a donc rien à voir a priori avec les questions religieuses, mais les répondantes étaient interrogées sur leur appartenance religieuse et les résultats donnent à réfléchir. Selon cette enquête, il est arrivé à 33\% des Françaises d'avoir au cours de leur vie un rapport sexuel avec une autre personne que leur partenaire, et ce serait le cas de $29 \%$ des catholiques pratiquantes et de $41 \%$ des protestantes ; les protestantes françaises seraient donc moins fidèles à leur conjoint non seulement que les catholiques pratiquantes (notons que les protestantes sont trop peu nombreuses pour que l'on puisse distinguer entre pratiquantes et non pratiquantes comme cela a été fait pour les catholiques; sans doute les chiffres auraient-ils alors été un peu différents), mais encore que la population globale. Moindre valorisation de la fidélité en protestantisme? plus grande honnêteté dans les réponses? effets liés à des différences d'appartenance sociale? A ce stade, le chercheur ne peut que s'interroger. Quand il lit par ailleurs dans la question suivante que $19 \%$ des catholiques pratiquantes en couple auraient eu $<\mathbf{6 0 1}>$ au moins un rapport sexuel avec une autre personne que leur partenaire actuel, pour 16 $\%$ de l'ensemble des Françaises, il ne peut que s'interroger à nouveau : serait-ce l'effet du refus du divorce en catholicisme qui conduirait à choisir l'adultère là où d'autres choisiraient le divorce ? et quelle est la cohérence des réponses à cette question avec la précédente ? Et le chercheur regrettera qu'il n'y ait, sur cette seconde question, pas de données concernant les protestantes, sans doute parce que les effectifs étaient trop petits pour que les pourcentages soient significatifs. On est, avec cet exemple, dans le cas d'une enquête qui peut servir à nourrir la réflexion préliminaire nécessaire à la construction d'un questionnement.

À ce stade, il s'agit donc surtout de noter des étonnements, de formuler de nouvelles questions et d'ouvrir des pistes d'explication, plutôt que d'élaborer des réponses définitives !

\section{Interpréter théologiquement et jouer la partition}

Pour le théologien, la tâche d'interprétation ne fait d'une certaine façon que commencer : avant de songer à proposer des pratiques, il lui faut encore effectuer ce travail de corrélation que nous avons évoqué en première partie. Ceci en marchant sur la ligne de crête que constitue la rencontre de deux convictions qui peuvent paraître contradictoires :

- La première est que l'Évangile ne saurait se réduire à nos désirs ; nos pratiques et nos opinions sont souvent une image du Dieu que nous nous construisons, davantage que du Dieu de Jésus-Christ - et cette affirmation ne vaut pas seulement pour nos opinions ou pratiques individuelles, mais aussi pour celles venues de la tradition chrétienne. De tous nos instincts, l'instinct religieux est celui qui a la plus besoin d'être évangélisé, disait volontiers Wilfred Monod, et c'est bien la tâche de la théologie pratique.

- Mais il faut en même temps refuser une attitude de soupçon systématique. Devant des opinions ou pratiques inhabituelles en Église, il faut commencer par se demander dans quelle mesure ces positionnements, même hétérodoxes, ne seraient pas une juste façon, pour les personnes, de traduire le message de l'Évangile dans les situations concrètes qui sont les leurs - ces situations dont justement les enquêtes ont pu aider à comprendre comment elles influaient sur les positions des personnes. Il s'agit finalement de faire une

\footnotetext{
16 « Les Françaises et l'infidélité féminine à l'heure des sites de rencontre », enquête IFOP du 8 janvier 2016
} 
certaine confiance à la capacité des personnes à théologiser - ce à quoi invite d'une certaine façon la conviction protestante du sacerdoce universel.

$<\mathbf{6 0 2}>$ Cette double conviction exigera du théologien qu'il soit prêt à remettre son ouvrage sur le métier, en réinterrogeant théologiquement les pratiques ecclésiales aussi bien que les opinions des répondants. Il lui faudra donc se demander quels sont les enjeux théologiques et/ou ecclésiologiques de tel ou tel positionnement, mais aussi quels en sont les enjeux humains. Et peut-être une réflexion sur ces enjeux permettra-t-elle de poser les questions un peu différemment.

Prenons à nouveau quelques exemples rapides :

L'enquête sur la prédication déjà citée a donné de forts indices pour penser que cet élément du culte joue en protestantisme le rôle d'un rituel chargé de rendre Dieu présent - un peu comme la célébration de l'eucharistie en catholicisme -, d'une façon presque indépendante de ce qui est dit : le fait qu'elle ait eu lieu semble en effet compter autant ou davantage que son contenu, dont les auditeurs ne se souviennent guère. Se pose alors la question d'une évaluation théologique de cette dimension rituelle - évaluation qui devra prendre en compte les apports de l'anthropologie sur le rôle du rite. Ce n'est qu'à la lumière de ces réflexions que le praticien pourra s'interroger sur les différentes approches possibles : faut-il tenter de casser le rite ? fautil plutôt essayer de mettre le besoin de rituel au service du message évangélique ? et alors comment? Et ce sera à chaque acteur de s'emparer de ces réflexions pour choisir ce qui lui paraît convenir le mieux pour son Église locale et pour lui-même.

Quant aux positions touchant à la guérison que nous avons elles-aussi déjà évoquées, elles renvoient bien sûr au cœur de la théologie, puisqu'elles posent fondamentalement la question centrale de savoir qui est Dieu et comment il intervient dans nos vies. Le théologien pratique pas plus que le systématicien, d'ailleurs - ne peut jamais avoir la prétention de trancher une telle question. Sa tâche pourra être de réfléchir sur les effets de telle ou telle conviction sur les personnes, et une nouvelle enquête pourrait là se révéler utile. Elle pourra être aussi de faire prendre conscience aux différents acteurs de l'inévitable pluralité à ce propos et de la richesse que peut constituer une telle pluralité si l'on veut bien sortir des anathèmes sur ceux qui ne pensent pas de la même façon.

On l'aura compris : la démarche de théologie pratique donne souvent l'impression d'être un long détour là où l'on aurait envie, parfois, de proposer tout de suite des recettes. Mais ce détour est indispensable pour que les pratiques ecclésiales puissent être vraiment enracinées dans l'Évangile et pertinentes pour nos réalités humaines. Dans ce détour, les enquêtes tiennent souvent une place importante - tout en n'étant elles-mêmes qu'une étape du chemin. Encore faut-il, pour qu'elles puissent jouer pleinement leur rôle, en respecter les règles.

\section{<603> CONCLUSION : LA QUESTION DE LA NORMATIVITÉ}

Ce qui m'amène à revenir, en guise de conclusion, sur une différence importante entre une enquête de sociologie pure et une démarche de théologie pratique, celle du rapport à une norme.

Une enquête sociologique n'a jamais de visée normative. Le sociologue n'est pas amené à dire ce qui est bien ou mal, ce qu'il faut faire ou ne pas faire ; son rôle est d'étudier les réalités et d'en comprendre les ressorts. La perspective du théologien pratique est différente. Il se réfère à une Église, à une tradition, à un corpus de textes qui induisent d'une certaine façon une dimension normative. Et il lui faudra souvent, in fine, suggérer des orientations concrètes, en tenant compte à la fois de l'état du réel manifesté par l'enquête et des visées propres au monde ecclésial. 
Cette différence de positionnement doit être prise en compte à toutes les étapes du processus de l'enquête - le danger étant que la dimension normative inhérente aux Églises influe, souvent à l'insu des acteurs, aussi bien sur la préparation de l'enquête que sur les réponses. Ainsi, du côté des répondants, tout un chemin de mise en confiance est indispensable pour qu'ils soient bien convaincus que, dans une approche sociologique, la seule «bonne réponse » est celle qui correspond le mieux à ce qu'ils pensent. Garantir l'anonymat (y compris à travers la façon de rendre le questionnaire à l'enquêteur), préférer un questionnaire que chacun remplit seul à un entretien avec un enquêteur, peuvent aider à établir cette confiance, de même que la mise en avant d'un sociologue plutôt que d'un responsable d'Église, porteur même malgré lui de cette dimension normative.

Il s'agira aussi, pour ceux qui mènent l'analyse des données recueillies, de suspendre le jugement pour essayer de comprendre en profondeur les réalités. Et ceci même si l'enquête révèle des écarts entre la réalité et ce que les Églises voudraient qu'elle soit, comme c'est presque toujours le cas!

Le travail de théologie pratique vise souvent à réduire ces écarts, pour que les pratiques ecclésiales et les comportements des chrétiens ressemblent un peu plus à ce que nous croyons qu'ils devraient être en fonction de notre compréhension de la foi chrétienne. Mais je plaide ici, on l'aura compris, pour que, avant de chercher à réduire l'écart, le théologien pratique prenne le temps de l'interroger : cet écart ne pourrait-il pas être fructueux ? peut-il dire quelque chose de la réalité du Dieu de Jésus-Christ ? en quoi peut-il questionner nos pratiques ecclésiales qui ont si souvent tendance à se scléroser?

Il faut d'ailleurs se souvenir qu'en termes d'écart, celui qui est au cœur de la foi chrétienne a bien des choses à nous enseigner, lui qui s'est si souvent situé en grand écart avec les autorités religieuses de son temps. Qu'il l'ait fait pour renforcer les exigences liées à la religion (pensons par exemple au Sermon sur $<\mathbf{6 0 4}>$ la Montagne : « vous avez entendu qu'il a été dit, mais moi je vous dis ... », Mt 7,21-48) ou pour les contester (ainsi, concernant le sabbat, qui " a été fait pour l'homme et non l'homme pour le sabbat », Mc 2,27), la liberté souveraine avec laquelle Jésus a agi peut nous interroger; et ceci d'autant plus que son souci premier était toujours de se rapprocher de ceux que ces exigences de la religion tenaient à distance de Dieu. Nos pratiques ecclésiales ne jouent-elles pas aussi parfois ce rôle de repoussoir?

Une des chances de ce travail d'enquête serait peut-être de nous aider à changer de paradigme grâce à la compréhension nouvelle qu'il donne des réalités. La tendance commune à la plupart des institutions humaines est de poser de règles, des conditions d'appartenance, et de chercher à enfermer le réel dans des fonctionnements à respecter. La tâche du théologien pratique ne serait-elle pas plutôt d'essayer de comprendre et de montrer comment le Royaume de Dieu est déjà présent, d'une certaine façon, dans la grande diversité du réel ? 